\title{
Welcome to Volume I - Issue II of the Biostatistics and Epidemiology International Journal
}

\author{
Brian P Mangum \\ Fiji National University, Suva, Fiji
}

Correspondence: Brian P Mangum, Fiji National University, Suva, Fiji,Tel 6913202480, Email epidemiology.doc@gmail.com

Received: April 18,2018 | Published: July 01, 2018

Copyright@ 2018 Mangum. This is an open access article distributed under the terms of the Creative Commons Attribution License, which permits unrestricted use, distribution, and reproduction in any medium, provided the original author and source are credited.

\section{Dear Colleagues and Readers:}

In April 2013, I arrived on an emergency flight into a small Pacific island nation in the midst of a dengue outbreak. This island had once been the scene of some of the bloodiest battles of World War II; and was now the scene of an outbreak that was rapidly spiralling out of control. The disease was spreading rapidly, there had been fatalities, the extent of the disease on outer islands where mobile phone service and internet did not reach was unknown. In many regards, the epidemic was out of control as it overwhelmed the local medical and public health workforce. The call had gone out from both government as well as regional NGO partners for an epidemiologist to lead the local team of epidemiological technicians. At the time, I was a consultant medical epidemiologist and professor in the region, and was chosen as one of the epidemiologists available who had an interest in infectious diseases.

Within a week - thanks to the hard work, and resilient attitudes of the local public health and medical workforce-an efficient surveillance network consisting of local healthcare workers and public health practitioners had been re-established and strengthened; integrated control measures were being put into place using environmental techniques; public information in local languages on when to seek care, and when to stay home, to alleviate the overburdened hospitals and clinics, was being broadcast over radio, as well as in newspapers and leaflets; and finally, hospital-based testing had been streamlined. The unique skill-set of epidemiology had brought order to chaos.

It was with great pride that I watched the local epidemiology team now turn to understanding the nature of the epidemic, using the time-honed statistical tools that are also hallmarks of our trade. We soon found trends among patients in terms of host, agent, and environment - the analytical triad of our profession - which suggested that there was something more at work than just dengue. A small, but significant, percentage of patients did not fit the case definition of dengue agreed upon by our team, as well as world-renowned consultants from WHO and other agencies. A tropical paradise of azure blue waters and sand as white as sugar was now home to a new medical mystery; one that could only be answered using epidemiological methods.
Within a very short period of time, our suspicions had been confirmed - chikungunya was present in small, but growing, numbers, masquerading as dengue. New experts, new protocols, new trainings of local healthcare workers, new health education campaigns, all to deal with this new threat. We soon sharedour findings and made contact with key stakeholders across the region to disseminate what we had learned in terms of diagnosis and management.

All of this was made possible because of the tools of epidemiology; demonstrating once again, the ongoing vitality and ability of our discipline to respond to and address the growing threats of the Twentyfirst Century.

But there is a second, equally important lesson here. And that is the story of platforms that allow researchers, no matter where they are in the world, to share their findings. Open-access journals, such as Biostatistics and Epidemiology International Journal, provide researchers from all areas of the world the means to share important and ground-breaking studies no matter where they work. In the case of the dengue/chikungunya outbreak in a small corner of the Pacific, we were worked on a limited budget both during the investigation, as well as in our efforts to share our important findings. Indeed, during the outbreak, our funding sources was cut in half as the local currency was devalued. Had it not been for innovative and unique means of sharing our information outside of traditional channels, our findings would have been restricted to a very small audience.

We see emerging and re-emerging threats worldwide, not just in the jungles and beaches of the Pacific. Zika remains a threat in tropical and sub-tropical areas. New strains of drug resistance are a global threat in industrialised as well as developing nations. The ongoing threat of non-communicable diseases such as type 2 diabetes, cardiovascular disease, cancer, and more threatens to engulf us no matter where we live and work. Just this week, I read an article from Victoria, Australia, on what the popular press is referring to as a 'Flesh-eating epidemic.' Buruli ulcer, caused by Mycobacterium ulcerans, is generally only found in Africa, but has seen a $400 \%$ increase in the last four years in Victoria, with 275 new cases last year alone. Writing in the Medical Journal of Australia, Dr Daniel P. O'Brien of the local health authority 
states that researchers do not understand what is driving the epidemic, and that as of now it remains a mystery. A mystery that requires the tools of epidemiology if we are to understand it, how it is spread, and most importantly how to stop its spread.

Indeed, the future is bright for epidemiology, as well as for global platforms such as this, which allow us to rapidly share high-quality research done at the local level. And so, I welcome you to this latest issue of Biostatistics and Epidemiology International Journal, as we share world class science with real-world impact.

Warmest regards,

Dr Brian P. Mangum

Editor-in-Chief 